thermal conductivity of $K_{i i}$ such that the conducted flow of heat through an elemental area $d s$ having associated direction cosines $l_{i}$ is

$$
-\left(K_{i j} T_{, i}\right) l_{i} d s .
$$

The net rate of heat flow into a volume bounded by a closed surface " $s$ " then is

$$
\int_{s}\left(K_{i j} T_{, i}\right) l_{i} d s
$$

The surface integral may be transformed to a volume integral,

$$
\int_{.}\left(K_{i i} T_{, i}\right) l_{i} d s=\int_{0}\left(K_{i j} T_{, i}\right)_{, i} d v .
$$

It is therefore necessary to investigate the properties of the form

$$
\left(K_{i j} T_{, j}\right)_{, i} \text {. }
$$

Considering the case where

$$
\begin{gathered}
K_{i i}=K_{i i}(T), \\
\left(K_{i j} T_{, i}\right)_{, i}=K_{i i} T_{, i i}+\frac{d K_{i i}}{d T} T_{, i} T_{, i}
\end{gathered}
$$

$T_{, i i}$ and $T_{, i} T_{, j}$ are both symmetrical in $i$ and $j$, therefore only the symmetrical portion of $K_{i j}$ will matter in $\left(K_{i j} T_{, i}\right)_{, i}$.

Since the case of $K_{i i}=K_{i j}(T)$ applies to a large class of practical applications, it is important to note, that for this case the anti-symmetrical portion of $K_{i j}$ if it existed at all would not contribute to a first law of thermodynamic energy accounting.

\title{
A CONVERSE TO THE VIRTUAL WORK THEOREM FOR DEFORMABLE SOLIDS*
}

BY W. S. DORN (Aircraft Gas Turbine Development Department, General Electric Company) AND

A. SCHILD (Carnegie Institute of Technology)

1. Introduction. Consider a continuous body occupying a volume $V$ and bounded by a closed surface $S .{ }^{1}$ Any system of stresses $\sigma_{i i},{ }^{2}$ satisfying the equilibrium conditions for zero body forces

$$
\begin{aligned}
& \sigma_{i i, i}=0, \\
& \sigma_{i j}=\sigma_{i i},
\end{aligned}
$$

${ }^{*}$ Received July 27, 1955. A. Schild's participation was supported by a research grant from the National Science Foundation.

'It is assumed that the body is simply connected and that the surface $S$ is composed of a finite number of pieces of each possessing a continuously turning tangent plane. All of the functions will be assumed to possess as many continuous derivatives in $V$ and on $S$ as are necessary for the theorems which will be used later.

2The subscripts range over the values $1,2,3$ and repeated subscripts will be summed over the entire range. Subscripts following a comma denote partial differentiation with respect to Cartesian coordinates $x_{i}$, e.g., $\sigma_{i, i}=\partial \sigma_{i j} / \partial x_{i}$. 
everywhere in $V$, and a system of virtual displacements $u_{i}$ in $V$, must together satisfy the equation of virtual work

$$
\int \sigma_{i j} \epsilon_{i j} d V=\int u_{i} \sigma_{i j} n_{i} d S,
$$

where $n_{i}$ is the unit outward normal to the surface $S$, and where $\epsilon_{i i}$ are the strains derived from the displacements $u_{i}$ by

$$
\epsilon_{i j}=\frac{1}{2}\left(u_{i, i}+u_{i, i}\right) \text {. }
$$

In this note the following converse to the theorem of virtual work is proved:

If, for a symmetric tensor $\epsilon_{i j}$ given in $V$ and for a vector $u_{i}$ given on $S$, the virtual work equation (3) is satisfied for all equilibrium stresses $\sigma_{i j}$ (i.e., for all $\sigma_{i}$ satisfying Eqs. (1) and (2)), then the $\epsilon_{i i}$ are compatible strains and are derivable, as in Eq. (4), from displacements $u_{i}$ which on $S$ have the given boundary values.

This theorem is similar to earlier results of $\mathrm{R}$. V. Southwell ${ }^{3}$, and to some recent results of H. L. Langhaar and M. Stippes. ${ }^{4}$ In contrast to the papers quoted, however, no stress-strain relations are assumed here and our theorem is not limited to linear elasticity theory.

2. Stress functions. For each value of $i=1,2,3, \mathrm{Eq}$. (1) states that the divergence of a vector $a_{i}=\sigma_{i i}$ vanishes. Thus the vector may be expressed as the curl of another vector, so that

$$
\begin{aligned}
\sigma_{i j} & =A_{i j n, n}, \\
A_{i j n} & =-A_{i n i} .
\end{aligned}
$$

Then Eq. (1) is identically satisfied. From Eq. (2) we have

$$
\left(A_{i j n}-A_{i n n}\right)_{, n}=0 .
$$

By the same argument as above, it follows that

$$
\begin{gathered}
A_{i j n}-A_{i i n}=B_{i j n m, m}, \\
B_{i j n m}=-B_{i i m n}=-B_{i i n m} .
\end{gathered}
$$

Using the symmetries given by Eqs. (6) and (8), Eq. (7) may be solved for $A_{i \text { in }}$, giving

$$
A_{i j n}=\frac{1}{2}\left(B_{n i j m}+B_{i n m i}+B_{i j n m}\right)_{, m} .
$$

Therefore, by Eq. (5),

$$
\sigma_{i i}=\frac{1}{2}\left(B_{n i j m}+B_{i m n i}\right)_{, m n} .
$$

We now define

$$
P_{n i j m}=\frac{1}{2}\left(B_{n i j m}+B_{i m n i}\right) .
$$

Then

$$
\sigma_{i j}=P_{n i j m, m n}
$$

${ }^{3}$ Proc. Roy. Soc. A 154, 4-21 (1936); Stephen Timoshenko 60th anniversary volume, The MacMillan Co., 1938, p. 211.

${ }^{4} \mathrm{~J}$. Franklin Inst. 258, 371-382 (1954). 
where, by Eqs. (8) and (10), $P_{n i j m}$ has the symmetries

$$
P_{n i j m}=-P_{i n i m}=-P_{n i m i}=P_{. j m i} \text {. }
$$

Thus any set of equilibrium stresses $\sigma_{i}$, are derivable, as in Eq. (11), from stress functions $P_{n i j m}$ which satisfy the symmetry conditions (12).

The six independent components of $P_{n i j m}$ can be expressed in terms of a symmetric second order tensor ${ }^{5} T_{r,}$ which is a dual tensor of $P_{n i i m}$ :

$$
T_{r .}=\frac{1}{4} \epsilon_{i r n} \epsilon_{i s m} P_{n i i m}, \quad P_{n i j m}=\epsilon_{i r n} \epsilon_{i s m} T_{r .},
$$

where $\epsilon_{i r n}$ is the usual completely skew-symmetric pseudotensor $\left(\epsilon_{123}=1\right)$. The six components of $P_{n i j m}$ or $T_{r}$ can also be identified with the stress functions $\chi_{1}, \chi_{2}, \chi_{3}$ of Maxwell and $\psi_{1}, \psi_{2}, \psi_{3}$ of Morera as follows: ${ }^{6}$

$$
\begin{array}{ll}
\chi_{1}=-P_{2323}=T_{11}, & \psi_{1}=2 P_{3112}=-2 T_{23}, \\
\chi_{2}=-P_{3131}=T_{22}, & \psi_{2}=2 P_{1223}=-2 T_{31}, \\
\chi_{3}=-P_{1212}=T_{33}, & \psi_{3}=2 P_{2331}=-2 T_{12} .
\end{array}
$$

For plane stress, the only non-vanishing stress function, $\chi_{3}=-P_{1212}=T_{33}$, reduces to Airy's stress function.

3. Compatibility equations. We shall now prove the first part of the theorem stated at the end of the Introduction, i.e., that the given stresses $\epsilon_{i j}$ are compatible.

Expressing the equilibrium stresses $\sigma_{i j}$ in terms of the stress functions $P_{n i i m}$, Eq. (3) becomes

$$
\int P_{n i j m, m n} \epsilon_{i j} d V=\int u_{i} P_{n i i m, m n} n_{i} d S .
$$

Applying Green's theorem twice to the volume integral, we obtain

$$
\int P_{n i j m} \epsilon_{i j, n m} d V=\int\left[u_{i} P_{n i j m, m n}+P_{n i m i \epsilon_{i m, n}}-P_{i i n m, m} \epsilon_{i n}\right] n_{i} d S .
$$

This equation must be valid for any equilibrium stresses, and thus for an arbitrary choice of the stress functions $P_{n i m}$. Let $P_{n i i m}$ vanish identically outside of a small volume surrounding an interior point $P$ of $V$, and let $P_{n i i m}$ be essentially constant inside the small volume. Then the surface integral on the right side of Eq. (16) vanishes. Since the $P_{n i \text { im }}$ at $P$ are still arbitrary except for the symmetry conditions (12), it follows that

$$
\epsilon_{i, m n}-\epsilon_{n i, i m}-\epsilon_{i m, i n}+\epsilon_{n m, i i}=0
$$

at any point $P$ in $V$. By continuity, these equations are also valid on $S$.

The equations (17) are the compatibility equations for strains and are necessary and sufficient conditions for the existence of a set of displacements $U_{i}$ in $V$ such that

$$
\epsilon_{i i}=\frac{1}{2}\left(U_{i, j}+U_{i, i}\right) \text {. }
$$

${ }^{5}$ C. Weber, Z. f. Ang. Math. und Mech. 28, 193-197 (1948).

${ }^{6}$ A. E. H. Love, $A$ treatise on the mathematical theory of elasticity, 4th ed., Dover Publications, 1944, p. 88. 
It remains to prove that these displacements may be so chosen, that they take on the given boundary values on $S$. This will be done in the next section.

4. Converse to the virtual work theorem. From Eq. (18), the (direct) theorem of virtual work for any equilibrium stresses follows by Green's theorem:

$$
\int \sigma_{i j} \epsilon_{i j} d V=\int U_{i} \sigma_{i j} n_{j} d S .
$$

Comparing with Eq. (3), we obtain

$$
\int\left(u_{i}-U_{i}\right) T_{i} d S=0, \quad T_{i} \equiv \sigma_{i j} n_{i} .
$$

This equation must hold for any choice of tractions $T_{i}$ on $S$ which are obtainable from a distribution of equilibrium stresses $\sigma_{i i}$ in $V$. It is well known that any distribution of surface tractions $T_{i}$ which is in static equilibrium can be so obtained.

Let $Q$ and $Q^{\prime}$ be any two points on $S$. Choose $T_{i}$ to be identically zero outside of two small areas $d S^{Q}$ and $d S^{Q^{\prime}}$ which respectively surround the points $Q$ and $Q^{\prime}$. Inside these areas, let the tractions be essentially constant and such that the forces

$$
F_{i}^{\circ}=T_{i}^{\circ} d S^{\circ}, \quad F_{i}^{Q^{\prime}}=T_{i}^{Q^{\prime}} d S^{Q^{\prime}}
$$

are equal and opposite and act in the direction of the line joining $Q Q^{\prime}$ :

$$
F_{i}^{0}=-F_{i}^{0^{\prime}}=\lambda\left(x_{i}^{0}-x_{i}^{0^{\prime}}\right), \quad(\lambda \neq 0) .
$$

This system of tractions is in static equilibrium. Equation (20) now gives

$$
\left[\left(u_{i}-U_{i}\right)_{Q}-\left(u_{i}-U_{i}\right)_{Q^{\prime}}\right]\left(x_{i}^{Q}-x_{i}^{Q^{\prime}}\right)=0 .
$$

This equation is equivalent to the geometric statement that the (infinitesimal) displacement $u_{i}-U_{i}$ leaves unchanged the distance between $Q$ and $Q^{\prime}$. Since this result holds for any two points on the closed surface $S$, it follows that $u_{i}-U_{i}$ must be a rigid body displacement:

$$
\begin{gathered}
u_{i}=U_{i}+\omega_{i j} x_{i}+\omega_{i}, \\
\omega_{i j}=-\omega_{j i}=\text { const., } \quad \omega_{i}=\text { const. }
\end{gathered}
$$

Equation (23) holds on the surface $S$ where the $u_{i}$ are given. We now use Eq. (23) to define throughout the volume $V$ a displacement $u_{i}$. It is then clear that this displacement takes on the assigned boundary values on S. From Eqs. (18) and (23) we also have

$$
\epsilon_{i j}=\frac{1}{2}\left(u_{i, j}+u_{i, i}\right) .
$$

This completes the proof of the converse to the virtual work theorem.

5. Conclusion. The converse to the virtual work theorem might be used in some problems on deformable solids, with given surface displacements $u_{i}$, to find among the members of an $N$ parameter family $\epsilon_{i j}\left(x_{1}, x_{2}, x_{3} ; \alpha_{1}, \alpha_{2}, \cdots, \alpha_{N}\right)$ of strain functions the one which is "least incompatible".

One could choose a set of $M$ equilibrium stresses $\sigma_{i i}^{1}, \cdots, \sigma_{i j}^{M}$ and determine the 
parameters $\alpha$ from the minimum principle

$$
\operatorname{Iin}_{\alpha_{1}, \cdots \alpha_{N}} \sum_{A=1}^{M}\left[\int \sigma_{i j}^{A} \epsilon_{i j} d V-\int u_{i} \sigma_{i j}^{A} n_{j} d S\right]^{2} .
$$

Alternatively, the parameters $\alpha$ could be determined from a minimax principle, such as

$$
\operatorname{Min}_{\alpha_{1}, \cdots \alpha_{N}}\left\{\operatorname{Max}_{\beta_{1}, \cdots \beta_{M}}\left[\int\left(\sum_{A=1}^{M} \beta_{A} \sigma_{i i}^{A}\right) \epsilon_{i j} d V-\int u_{i}\left(\sum_{A=1}^{M} \beta_{A} \sigma_{i i}^{A}\right) n_{i} d S\right]^{2}\right\},
$$

where the parameters $\beta$ must satisfy $\sum_{A=1}^{M} \beta_{A}^{2}=1$.

\section{A NOTE ON LAMINAR AXIALLY SYMMETRIC JETS*}

BY MARK BERAN (Wellesley, Mass.)

Summary. It is shown that there is no stream function of the form $\psi=r f(\theta)$, that is compatible with the complete Navier-Stokes equations, which represents a jet issuing from a small circular hole in an axially symmetric cone.

The asymptotic velocity field of a laminar viscous jet is generally accepted to have a stream function of the form $\psi=r f(\theta)$, corresponding to self-similar flow (Schlichting [1], Squire [2], and Yatseev [3]). The authors referred to have based their discussion on the fact that this assumption of self-similarity is compatible with both the boundary layer equations, and with the full Navier-Stokes equations.

The purpose of this note is to establish a serious shortcoming of such models. It is shown that there is no continuously differentiable velocity field associated with a stream function of the form $\psi=r f(\theta)$, which satisfies the Navier-Stokes equations and also adheres to a conical wall $\theta=\alpha>0$.

Specifically, if $\psi=r f(\theta)$, then the velocity components in the $r$ and $\theta$ directions are respectively [4]

$$
\begin{gathered}
u_{r}=\left[\frac{1}{r \sin \theta}\right] \frac{d f}{d \theta}, \\
u_{\theta}=\left[\frac{-1}{r \sin \theta}\right] f .
\end{gathered}
$$

The Navier-Stokes equations are equivalent to [5]

$$
f^{2}=4 \nu \cos \theta f-2 \nu \sin \theta \frac{d f}{d \theta}-2\left(c_{1} \cos ^{2} \theta+c_{2} \cos \theta+c_{3}\right)
$$

for suitable constants $c_{1}, c_{2}, c_{3}$. We shall show that there is no solution of (3) which (i) makes $u_{r}$ and $u_{\theta}$ continuous for $r>0$, and (ii) satisfies $u_{r}(\alpha)=u_{\theta}(\alpha)=0$, for 0 $<\alpha \leq \pi$.

To show this, we also consider the differentiated form of (3), which is

$$
\frac{-f}{\sin \theta} \frac{d f}{d \theta}=2 f-2 \sin \theta \frac{d}{d \theta}\left[\frac{1}{\sin \theta} \frac{d f}{d \theta}\right]-\left(2 c_{1} \cos \theta+c_{2}\right) \text {. }
$$

*Received June 28, 1955; revised manuscript received October 5, 1955. The work on this paper was partly supported by Contract N5ori-07634 with the Office of Naval Research. 